\title{
http://dx.doi.org/10.21611/qirt.1994.037 Edge-effects and defect sizing by transient thermography
}

by ALMOND D. P.*, SAINTEY M .B.** and LAU S. K.*

" School of Materials Science, University Of Bath, Bath, Avon, BA2 7AY. UK.

** TWI, Abington Hall, Abington, Cambridge, CB1 6AL. UK.

\section{Abstract}

Thermal edge-effects for crack-like defects have been calculated using the Wiener-Hopf technique. These lead to an experimentally verified prediction that transient thermographic defect images shrink systematically with elapsed time. A simple method of defect sizing is proposed based on accounting for defect shrinkage. Finite difference modeling is used to investigate the dependence of image shrinkage on defect size and depth.

\section{Introduction}

The principle employed to obtain an image of a sub-surface defect by transient thermography is deceptively simple. A surface is heated by powerful flash lamps and subsequent thermal transients are recorded by an infrared camera. Defects cause perturbations in heat flow which are revealed by the camera. Whilst there is now a considerable body of practical experience of the application of the technique, there is rather less precise quantitative information about the image formation process that could lead to reliable defect sizing. In an earlier paper [1] the authors considered circular air gap defects of differing radii by treating them as buried uniformly heated disks; a treatment similar to that recently employed by Thomas et al [2] as an inversion technique for thermal wave images. This work produced computed images which exhibited the principal features of those obtained experimentally but it was noted that its quantitative value was limited because defect edgeeffects were not incorporated. This deficiency was rectified analytically in two recent papers $[3,4]$ in which the improved analysis was shown to lead to a simple means of defect sizing. Edge-effects are discussed further in this paper and some finite difference work is presented which expands on the analytical results.

\section{Edge-effects}

The thermal edge-effect occurring at the tip of a perfect crack-like defect may be obtained analytically by adapting the well established Wiener-Hopf [5] solution for the scattering of light or sound from the edge of a semi-infinite half-plane. Whilst in transient thermography a time domain solution is required, it is analytically convenient to first solve the problem in the frequency-domain, i.e. to obtain a thermal wave solution, and then to construct a time-domain solution by a suitable transformation. In the frequency-domain, we consider the problem of a crack at a set depth beneath the surface of a semi-infinite solid, in a plane parallel to the surface extending in the positive $x$ direction from zero to infinity, subjected to a periodically modulated thermal flux incident normal to the crack surface. This flux generates thermal waves in the solid having complex wave numbers $\sigma=(1+i) / \mu$, in which $\mu$ is the thermal diffusion length, $\mu=(2 \alpha / \omega)^{1 / 2}$, where $\alpha$ is the thermal diffusivity and $\omega$ is the angular frequency of thermal modulation. The resulting Wiener-Hopf solution for the temperature profile along the upper surface of the crack is shown in figure 1 plotted as a function of reduced distance, $x /$ $\mu$, from the crack edge. This figure shows the excess temperature, or temperature contrast, caused by the presence of the crack. In the earlier work $[1,2]$ this had been taken to be a "tophat" profile rising abruptly from zero to a constant value across the whole surface of the crack. The new analysis shows an edge-effect amounting to a decay in temperature contrast over a 
http://dx.doi.org/10.21611/qirt.1994.037

distance of about a thermal diffusion length from the edge of the crack. A crucial feature of the edge-effect is the decay of thermal contrast to zero at the crack tip. This, and the edgeeffect as a whole, is caused by the flow of heat around the crack tip from the hot upper surface of the crack to the cold under surface. The symmetry of this process ensures that there is no net flux increase for material in front of the crack tip. The earlier work effectively ignored the cold side of the crack.

\section{Defect images}

Although the edge-effect shown in figure 1 is only rigorously valid for an infinite straight edged crack, it should also provide a reasonable approximation to the edge-effects at real cracks of moderate curvature. In the recent papers $[3,4]$ it was shown that the profiles of defect images produced at the surface by different frequency thermal waves, computed using the temperature distribution shown in figure 1 , intersect at a common point as shown in figure 2. At this point the amplitude is exactly half that at the image center. For a $10 \mathrm{~mm}$ diameter defect this point occurred at a distance $0.28 \mu$ within the true radius of the defect. Hence if the half-amplitude points of an image were used to measure defect size, it was predicted that the result for a $10 \mathrm{~mm}$ diameter defect would be to underestimate the defect radius by $0.28 \mu$. This reduction in apparent size is a consequence of the edge-effect. If a top-hat thermal profile is employed the computed image profiles also cross at haif-amplitude but precisely over the crack edge.

\section{Transient solution}

The flash excitation employed in transient thermography may be modeled as a Dirac-delta function like excitation which results in a continuous spectrum of Fourier components. Formally, the time-domain response of a solid may be obtained by a Laplace inversion of the frequency-domain response. However, it has been found $[6,7]$ that the transient response at a particular time $t$ after a flash is, to an approximation, that which would be computed for a thermal wave with an effective thermal diffusion length $\mu_{\text {eff }}=2(\alpha t)^{1 / 2}$. In transient thermography this amounts to interpreting images obtained at increasing times after the flash as being formed by thermal waves of increasing thermal diffusion length. As the underestimation in defect radius is $0.28 \mu$, it is therefore predicted that defect radius, taken to be at half peak image amplitude, should shrink with elapsed time.

\section{Comparison with experimental data}

A set of half-amplitude measurements of the diameters of transient thermography images of a $10 \mathrm{~mm}$ diameter defect in mild steel [1] (FWHM full-width at half-maximum measurements) is shown plotted in figure 3 as a function of the square root of elapsed time after flash. An uncertainty of about $0.25 \mathrm{~mm}$, corresponding to an image pixel, must be added to each FWHM measurement. The predicted shrinkage with time is confirmed by the experimental data. The solid line in the figure is the theoretical shrinkage obtained assuming the thermal diffusivity of mild steel to be $14 \times 10^{-6} \mathrm{~m}^{2} / \mathrm{s}$. This line has a slope which is clearly close to that of the experimental data.

The magnitude of the shrinkage is large, underlining the danger of underestimating defect size, even when making use of video frames collected as soon as possible after the flash. However, there is a simple method of correcting for this shrinkage and of determining defect size. FWHM measurements of image size should extrapolate back to true defect size at zero time. If this procedure is applied to the data in the figure a defect diameter of $10.4 \mathrm{~mm}$ is obtained which amounts to a $4 \%$ overestimation of the defect's size. This small overestimation is considered to be far preferable to the serious underestimations that could result from using uncorrected data.

The above technique will fail for very small defects or at very long times where edgeeffects at opposite sides of the defect overlap. The latter occurs here at times greater than 
about 0.45 seconds after the flash and at longer times the shrinkage ceases. The behavior at longer times is outside of the scope of the Wiener-Hopf analysis. For this reason, and to check the analytical results, the same problem has been solved using fipite-difference modeling.

\section{Finite difference modeling}

The numerical results presented here were calculated using a PC based finite difference model. The model was formulated in cylindrical polar co-ordinates, using the alternating direction implicit (ADI) technique for the solution of the resulting finite difference equations. The modeled crack defects were circular, and parallel to the surface of the material in which they were embedded. The use of cylindrical polar co-ordinates $(r, z, \phi)$ allowed the problem to be reduced to 2 dimensions, assuming the thermal properties of the material to be isotropic and homogeneous. The symmetry of the problem about the $z$ axis eliminates the $\phi$ dependence enabling solution by the modeling of only the $(r, z)$ plane. The crack defect was modeled as a contact resistance between 2 layers of nodes, and was taken to have a value of $1.0 \mathrm{~m}^{2} \mathrm{~K} / \mathrm{W}$ (equivalent to an air gap $2.5 \mathrm{~cm}$ thick). Such a high value of contact resistance is unrealistic, but was used to simulate what might be termed a 'perfect crack' for comparison with the Wiener-Hopf solution. Convective heat losses have been neglected here for simplicity. All of the results presented here are for crack defects in mild steel, the thermal properties of which were taken to be: thermal conductivity $k=64 \mathrm{~W} / \mathrm{mK}$, density $\rho=7850$ $\mathrm{Kg} / \mathrm{m}^{3}$, and specific heat capacity $\mathrm{c}=580 \mathrm{~J} / \mathrm{KgK}$. The results are for Dirac-delta pulse heating of the samples; this was achieved by first calculating the thermal response of the sample to a step-on heating regime, and then differentiating the result with respect to time to evaluate the pulse heating solution.

Figure 4 shows the normalized crack surface temperature profiles calculated at times 0.1 , $0.3,0.5,0.7$ and 0.9 seconds after the flash heating of a sample containing a crack of radius $10 \mathrm{~mm}$, at a depth of $1 \mathrm{~mm}$. It can be seen that the temperature contrast on the crack surface falls almost to zero at the crack tip, as predicted by the Wiener-Hopf solution. At the shortest time, the profile is quite sharp, but softens with increasing time after the pulse. Figure 5 shows the upper surface image normalized temperature profiles at the same times as those shown in figure 4. The features are very similar to those of the crack surface profiles in that they are sharp at short times, and soften with increasing time. Figure 6 shows the variation of surface temperature profile FWHM with time for the first second after the heating pulse. The maximum temperature contrast over the crack occurs at approximately 0.53 seconds after the pulse, and is shown in the figure as a filled circle on the FWHM curve. It can be seen that if FWHM was taken as the measure of defect size, and was measured at the time of maximum contrast, an underestimate of $20 \%$ in the defect diameter would result. The reduction in the FWHM with time after the pulse is in agreement with that predicted by the analytical approach.

Figure 7 shows plots of the variation in FWHM for cracks of radius $5 \mathrm{~mm}$, at various depths below the surface. The depths considered were $0.25,0.5,1.0,1.5$ and $2.0 \mathrm{~mm}$. It is seen that the FWHM for a shallow crack reduces much more rapidly with time than for a deeper crack, making the sizing of defects very close to the surface a more difficult task than might be expected. Each FWHM curve has a filled circle marking the value of the FWHM at the time of maximum contrast. It is interesting to note that a measurement of the FWHM at the time of maximum contrast at all the depths would give almost the same value, approximately $8 \mathrm{~mm}$, a $20 \%$ underestimate in the true diameter of the crack.

Figure 8 shows plots of the FWHM for cracks of various diameters, all at a depth of $1 \mathrm{~mm}$. The diameters considered were 2, 4, 6, 8 and $10 \mathrm{~mm}$. The lowest curve is for the $2 \mathrm{~mm}$ diameter crack, and shows a behavior totally unexplained by the Wiener-Hopf solution. By the time of maximum contrast, once again shown in the figure by a filled circle, the FWHM has gone through a minimum, and is increasing. What has occurred is the edge effects from opposite sides of the crack have overlapped. This flattens and broadens the temperature contrast on the crack surface, which then causes a similar effect at the sample surface. All of the FWHM curves go through a minimum, but the time at which this occurs is dependent on the defect size, the larger defects reaching the minimum later. It seems in principle to be 
possible to use this time of minimum FWHM as a means of defect sizing, but in reality would be extremely difficult to measure. For small diameter cracks, the minimum is quite sharp, but occurs so soon after the heating pulse that there is very little surface temperature contrast over the crack at that time. For the larger defects, the minimum is shallow, and occurs after the contrast maximum.

\section{Conclusions}

The analytical study of the thermal edge effect at the tip of a crack has provided new insight into the temporal development of transient thermographic images of buried defects. It has led to the recognition that FWHM measurements relate to defect size and that the image of a defect shrinks in a systematic way with elapsed time. The form of this shrinkage provides a means of assessing the true size of a defect.

\section{Acknowledgments}

The authors are particularly grateful to Professor J.R.Willis for showing them the WienerHopf method provided a solution of their problem and for many helpful discussions about theoretical aspects of this work. The authors are also grateful to TWI and PowerGen for their support of this work.

\section{REFERENCES}

[1] LAU (S.K.), ALMOND (D.P.) and MILNE (J.M.), NDT \& E Int. 24, 195 (1991).

[2] THOMAS (R.L.), FAVRO (L.D.), CROWTHER (D.J.) and KUO (P.K.), Proc. Eurotherm Sem. 27 Quantitative Infrared Thermography, edited by D.Balageas, G.Busse and G.M.Carlomagno (Editions Europèennes Thermique et Industrie, Paris, 1992), p.278.

[3] ALMOND (D.P.) and LAU (S.K.), Appl.Phys.Lett. 62, 3369 (1993).

[4] ALMOND (D.P.) and LAU (S.K.), J. PHYS. D: Appl. Phys. 27, 1063 (1994).

[5] NOBLE (B.), Methods Based on the Wiener-Hopf Technique (Pergamon, London, 1958).

[6] KANSTAD (S.O.) and NORDAL (P.E.), Can.J.Phys. 64, 1155 (1986).

[7] LAU (S.K.), ALMOND (S.K.) and PATEL (P.M.), J.Phys D: Appl.Phys.24, 428 (1991). 
http://dx.doi.org/10.21611/qirt.1994.037

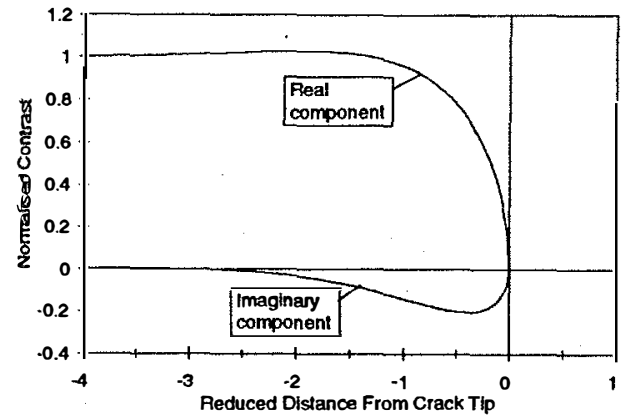

Figure 1: Real and imaginary components of the temperature contrast on the crack surface evaluated using the Wiener-Hopf technique.

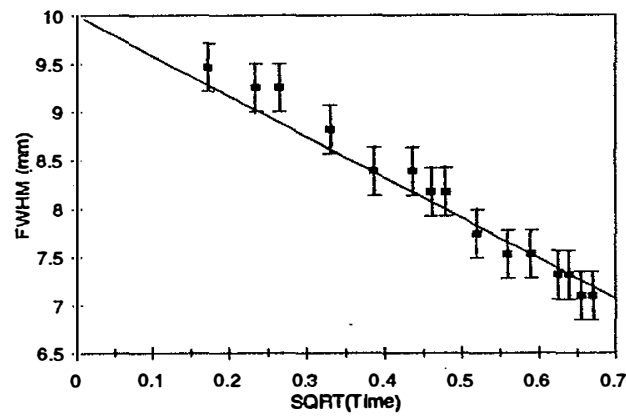

Figure 3: FWHM measurements taken during a transient thermography experiment, imaging a crack-like defect in mild steel. The solid line shows the prediction from the analytical treatment.

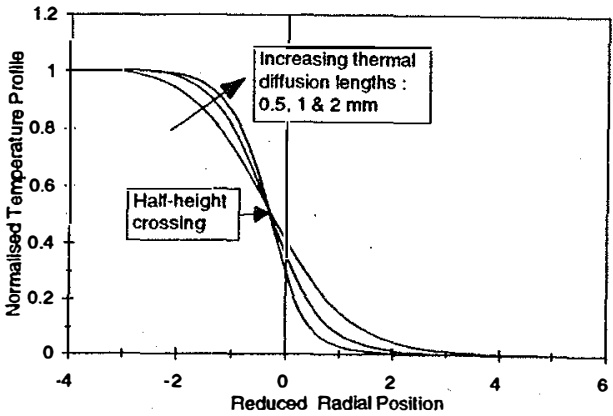

Figure 2: Upper surface temperature profiles of a $10 \mathrm{~mm}$ diameter crack defect in mild steel, imaged using thermal waves of various thermal diffusion lengths.

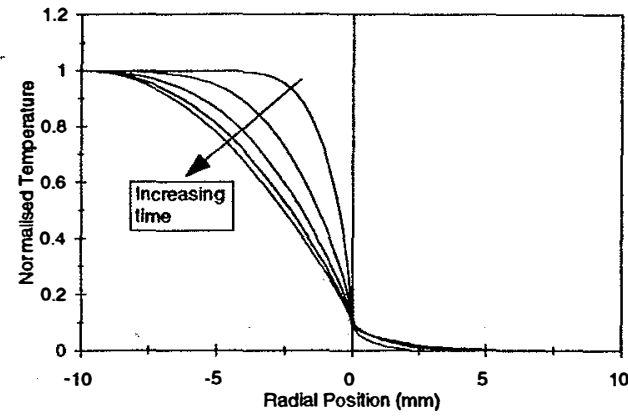

Figure 4: Normalised temperature profiles on the surface of a $10 \mathrm{~mm}$ radius crack at times $0.1,0.3,0.5,0.7$ and 0.9 seconds after the pulse heating. The profiles were calculated using the finite-difference model. 
http://dx.doi.org/10.21611/qirt.1994.037

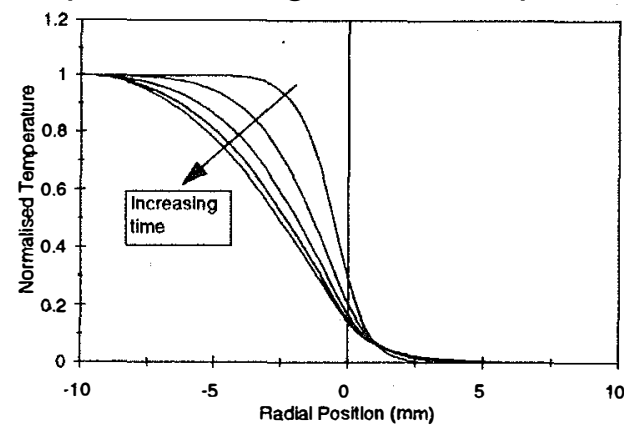

Figure 5: Normalised temperature profiles on the upper surface at times $0.1,0.3$, $0.5,0.7$ and 0.9 seconds after the pulse heating of a mild steel sample containing a $10 \mathrm{~mm}$ radius crack defect. The profiles were calculated using the finite-difference model.

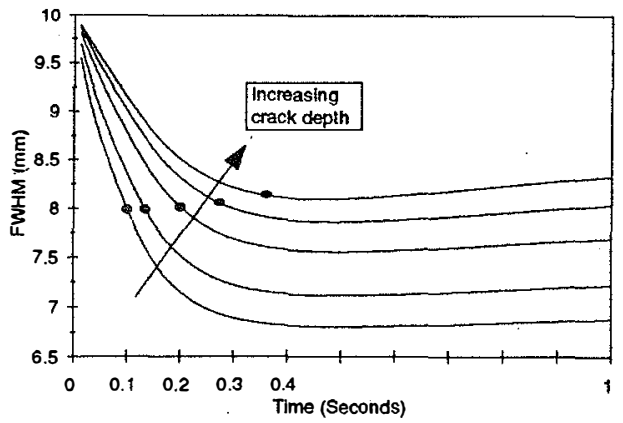

Figure 7: The variation of FWHM with time for $5 \mathrm{~mm}$ radius crack defects at depths $0.25,0.5,1.0,1.5$ and $2.0 \mathrm{~mm}$. The filled circles on the curves indicate the time at which maximum surface temperature contrast occurred.

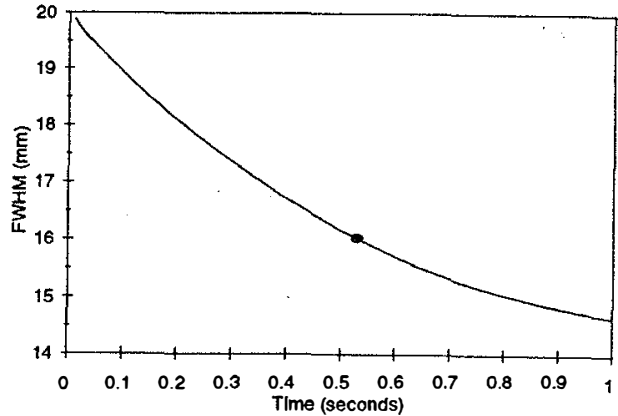

Figure 6: The variation of FWHM with time of the upper surface temperature profiles calculated for a mild steel sample containing a $10 \mathrm{~mm}$ radius crack.

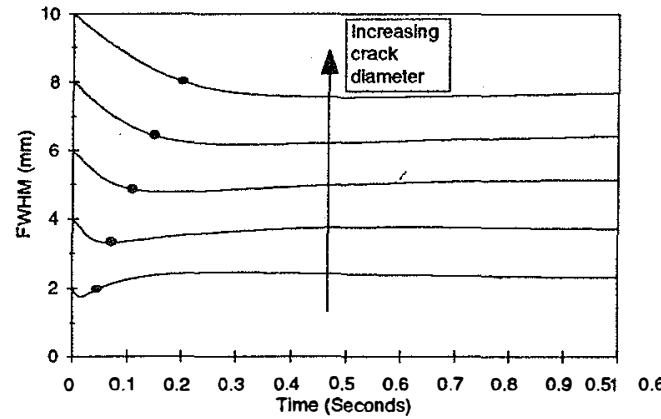

Figure 8: The variation of FWHM with time for various diameter crack defects all at a depth of $1 \mathrm{~mm}$. Once again the filled circles indicate the time at which the maximum surface temperature contrast occurred. 